\title{
Impacto del Programa de Hipertensión Arterial en Un Centro de Salud de Soledad, Atlántico (Col)
}

\section{Impact of Hypertension Program in a Health Center Soledad, Atlántico (Col)}

\author{
Arrieta-Reales $N^{1}$, Blanquicet Jimenez $R^{2}$, Borrero Varela $E^{2}$, Muños Vesga $E^{2}$ \\ ${ }^{1}$ Departamento Docencia - Servicio de las Ciencias de la Salud - Universidad Simón Bolívar, ${ }^{2}$ Programa de Medicina - Universidad Simón Bolívar
}

\begin{abstract}
Resumen
Objetivo: Evaluar el impacto del programa de Hipertensión Arterial del Centro de Salud Villa Estadio de Soledad - Atlántico, del 2009 al 2014. Materiales y métodos: Estudio correlacional, longitudinal y cuantitativo. Instrumentos: encuesta estructurada de preguntas cerradas, validado con el estadístico de Kuder-Richardson, confiabilidad del 0,80\%; la información de las historias clínicas tabulada en base de datos mediante Microsof Excel. La muestra, 62 pacientes con dos a cinco años de permanencia en el Programa de HTA.

Resultados: El análisis de los datos de las historias clínicas se utilizo Statgraphics Centurion XVI y Limesurvey; los resultados de la encuesta a través de gráficos por porcentaje de frecuencia. El Programa de HTA produjo modificación de los estilos de vida de los pacientes: cambios de las presiones sistólica y diastólica cuya significancia para la sistólica estuvo p: 0,0036 y la diastólica p: 0,0042; el 71\% disminuyeron y mantuvieron el peso en el rango ideal. El 60,5\% disminuyeron y mantuvieron los niveles de glicemia en rango normal. En el $71 \%$ de los pacientes se encontró una correlación positiva entre las variables peso y presión arterial sistólica y diastólica; igualmente el HDL que al encontrarse por encima de $50 \mathrm{mg} / \mathrm{dl}$ en el $60 \%$ de los pacientes se constituye en una relación positiva como factor protector del riesgo cardiovascular.

Conclusiones: Se probó la hipótesis que el programa de HTA del Centro de Salud Villa Estadio, produjo un impacto positivo en la modificación de los estilos de vida de los pacientes.
\end{abstract}

Palabras Claves: Impacto; Programa de Hipertensión Arterial; Estilos de Vida (Fuente: DeCS).

\footnotetext{
Abstract

Objective: Evaluate the impact of the program Hypertension Health Center Villa Estadio Soledad - Atlántico from 2009 to 2014.

Materials and methods: Correlational, longitudinal and quantitative study. Instruments: structured survey of closed questions, validated

Correspondencia: Nancy Arrieta Reales. Programa de Medicina, Facultad de Ciencias de la Salud. Universidad Simón Bolivar. Cr 59 \# 59 - 65. Barranquilla, Atlántico, Colombia. mmendinueta@unisimonbilivar.edu.co.

Citar: Arrieta-Reales N, Blanquicet Jimenez R, Borrero Varela E, Muños Vesga E. Impacto del Programa de Hipertensión Arterial en Un Centro de Salud de Soledad, Atlántico (Col). Cienc e Innovación en Salud [Internet]. 2015 Jan 1;3(1):27-32. Available from:

http://publicaciones.unisimonbolivar.edu.co:82/rdigital/ojs/index.php/innovacionsalud/article /view/236/233.

Parte de este trabajo se encuentran soportado en la investigación titulada «Evaluación del Impacto del Programa de Hipertensión Arterial en Un Centro de Salud de Soledad, Atlántico (Col)» tesis para obtener el título de Médico de la Universidad Simón Bolívar (Colombia).

Recibido: Oct. 12 de 2014 / Modificado: Nov. 11 de 2014 / Aceptado: Nov. 21 de 2014.
}

statistical Kuder-Richardson reliability of $0.80 \%$; tabular information from medical records in database using Microsoft Excel ${ }^{\circledR}$. The sample, 62 patients with two to five years in the HTA Programme Results: The analysis of data from clinical records and Limesurvey C Statgraphics Centurion $X V I^{\circledR}$ was used; the survey through graphics percentage frequency. The HTA program produced modification of the lifestyles of patients: changes in systolic and diastolic to systolic whose significance was p: 0.0036 and diastolic p: $0.0042 ; 71 \%$ decreased and kept the weight in the ideal range. $60.5 \%$ and decreased blood glucose levels remained in the normal range. In $66 \%$ of patients a positive correlation between the variables weight and systolic and diastolic blood pressure was found; HDL also found that above $50 \mathrm{mg} / \mathrm{dl}$ in $60 \%$ of patients constitutes a positive relationship as a protective factor in cardiovascular risk.

Conclusions: The hypothesis that the HTA program Health Center Villa Estadio, had a positive impact in changing the lifestyles of patients tested.

Keywords: Impact, Hypertension Program, Lifestyle (Source: MeSH, NLM).

\section{INTRODUCCIÓN}

La Hipertensión Arterial (HTA), es un problema prioritario de salud pública de altísimo costo social y sanitario, se atribuye al aumento de la población, a su envejecimiento y a factores relacionados con su comportamiento (dieta mal sana, uso abusivo de alcohol, obesidad, escasa actividad física, entre otros). Se ha evidenciado que un IMC de 25 , el riesgo de prehipertensión se incrementa entre un 11 a $15 \%$ por cada $\mathrm{kg} / \mathrm{mt} 2$ ganado (1)(2)(3). Así mismo han encontrado que el sexo más afectado con HTA es el femenino en un $74 \%$ y el sedentarismo se encuentra presente en la mayoría de los pacientes hipertensos (4)(5)(6).

Estudios han demostrado que: una reducción de sodio puede disminuir $3,9 \mathrm{~mm} / \mathrm{Hg}$ la presión sistólica y 1,9 $\mathrm{mm} / \mathrm{Hg}$ la presión diastólica (7); la obesidad se constituye en uno de los factores de riesgo para el desarrollo de diabetes tipo 2, enfermedades cardiovasculares, hipertensión arterial, dislipidemias, osteoarticulares, entre otros (8); y, el ejercicio reduce la 
presión arterial sistólica y la diastólica, produciendo una disminución de la hipertensión arterial leve y moderada, sin el uso de medicamentos y acompañado de una dieta balanceada, reduciéndose la incidencia de hipertensión a valores superiores al $50 \%(9)(10)(11)$.

La HTA, representa uno de los trastornos crónicos más frecuentes en el mundo occidental en mayores de 25 años y es la primera causa de muerte en Colombia (12). El Gobierno Nacional, ha fijado para el 2021 se reduzca en un $25 \%$ a partir de la línea de base definida en el año 2014 (13). El Ministerio de Salud y Protección Social ha orientado las intervenciones para garantizar su efectividad descritos en el Plan Decenal de Salud Pública (PDSP) Colombia 2012-2021, teniendo como objetivos, "favorecer de manera progresiva y sostenida la reducción a la exposición a los factores de riesgo modificables en todas las etapas del transcurso de vida; generar condiciones y fortalecer la capacidad de gestión de los servicios, para mejorar la accesibilidad y la atención integral e integrada de las Enfermedades No Transmisibles» (14).

Se constituye la HTA, en uno de los principales motivos de consulta médica y de prescripción farmacológica, su prevalencia es alta en un $25 \%$, con un total de mil millones de hipertensos (15). Hacia el 2010, 691 millones de personas sufrían de hipertensión arterial en el mundo, cifra que va en incremento y en 20 años habrá aumentado más del 50\%. La HTA aumenta con la edad y existe una alta probabilidad que las personas después de los 50 años la padezcan, a causa del aumento de la resistencia periférica y de la rigidez arterial propios del envejecimiento (16-18).

Un estudio que evaluó el impacto de un programa para la prevención de enfermedades cardiovasculares 2005-2006 en Santander, demostró con las variables de edad, peso, genero, tensión arterial, glicemia, colesterol total, HDL, LDL, triglicéridos, glicemia, número de consultas por año y estrato socioeconómico, que el programa tubo mejores resultados en los pacientes de género masculino y en menores de 60 años (19). De igual manera el estudio efectuado en el hospital San Ignacio evidencio que el $87 \%$ de los pacientes asistentes al programa de HTA mantuvieron los niveles de presión arterial inferior a 140/90 (20).

Así mismo en la encuesta realizada a la población de Soledad-Atlántico, mostró que la hipertensión arterial con un $15,4 \%$, ocupaba el primer lugar entre las patologías que más demanda una atención médica, seguida de la migraña con $8.9 \%$ y el colesterol con 8,2 $\%$; encontrándose diferencias por sexo con un $16,7 \%$ para hombres y $12,7 \%$ para mujeres (21).
Otros aspectos de importante relevancia integran: ala realización de buenos controles por el equipo interdisciplinario de salud, que en Estados Unidos, el grado de buen control es de solo un $29 \%$ y en los países latinoamericanos la situación es más complicada, los porcentajes de pacientes con un adecuado control son mucho más bajos. b- La educación brindada a los pacientes, en un estudio quedó demostrado que la intervención educativa de enfermería produjo una disminución en la mortalidad, en el número de reingresos y en los costos de manejo de los pacientes $(22-25)$

Los programas educativos se constituyen en una estrategia que promueve la enseñanza del autocuidado, hacia la generación de un cambio de comportamiento, orientados al logro de los objetivos terapéuticos, mejorar y mantener la calidad de vida, impedir las complicaciones y muertes, promoviendo la autonomía y el empoderamiento del paciente (26). A su vez, requiere de la disponibilidad adecuada de medicamentos y controles periódicos en los cuales realicen los ajustes necesarios a la medicación. Esos factores generan un impacto positivo en la reducción de las complicaciones cardiovasculares y garantizan la adherencia al tratamiento farmacológico y no farmacológico $(27,28)$.

Se debe tener presente que estos programas dirigidos a incrementar los niveles de adherencia, integran condiciones con multicomponentes con una amplia variedad de conductas y habilidades en diferentes grados de complejidad, requiriendo de la participación activa del paciente $(29,30)$.

A pesar de la importancia de los programas de HTA, al revisar la bibliografía existente se encontraron pocos estudios a nivel a nivel de Colombia y del Atlántico, que evaluaran su impacto. Teniendo presente que se constituye en una forma sistemática para mejorar y dar cuenta de las acciones de salud pública mediante la participación de los procedimientos que son útiles, factibles, éticos y precisos (31). En este sentido, surgió el siguiente interrogante: ¿Cuál es el impacto del Programa de HTA del Centro de Salud Villa Estadio de Soledad Atlántico del 2009 - 2014?.

\section{MATERIALES Y MÉTODOS}

Estudio correlacional, longitudinal y cuantitativo. Se obtuvo la información de las historias clínicas de los paciente que tuvieran cinco años de permanencia en el programa del 2009 al 2014. La muestra estuvo constituida por 62 pacientes que cumplían con el criterio de inclusión ya expreado. Se analizó el estado clínico de los pacientes al ingreso, en un control intermedio y en el 
último, en cuanto a las variables: peso, presión arterial sistólica, presión arterial diastólica, niveles de glicemia, niveles de lípidos y función renal (Bun y Creatinina).

El instrumento fue una encuesta diseñada de acuerdo a las variables del estudio: edad, sexo; cambios en la salud desde que asisten al programa; si han recibido educación; aplicación de hábitos pocos saludables; modificación de hábitos poco saludables por la educación que han recibido; cambios en su condición física; y, satisfacción por el programa. Para la validación del instrumento se aplicó el estadístico de kuderrichardson $\left(\mathrm{kr}_{20}\right)$, arrojando una confiabilidad de 0,80.

Las técnicas para el análisis de los datos fueron descriptivas y se correlacionaron los resultados entre las variables; la correlación realizada fue de Spearman de múltiples rangos. Los estadísticos aplicados para la medición de los niveles de significancia fueron Statgraphics Centurion $\mathrm{XVI}^{\circledR}$ y LimesurveyC. El análisis de los datos de la encuesta se realizó a través de gráficos por porcentaje de frecuencia.

\section{RESULTADOS}

El $82 \%$ de los pacientes pertenecían al sexo femenino y el $18 \%$ al masculino; el rango etario de los pacientes en mayor porcentaje los adultos mayores con un $45 \%$, seguido en edad de la senectud en un 34\%; en porcentajes menores adultos intermedios con el $14,5 \%$ y adultos jóvenes en un $9 \%$.

En cuanto a las condiciones de salud al ingreso de los pacientes (primer control) el 19,4\% era fumador; el $80,6 \%$ practicaba el sedentarismo; el 75,8\% consumía alimentos ricos en grasas y calorías y el $100 \%$ tenía niveles de presión arterial por encima de 140/90. Se evidenció una disminución de los niveles de las presiones sistólica y diastólica entre el primer y último control, cuyo nivel de significancia para la sistólica estuvo p: 0,0036 y la diastólica p: 0,0042; correspondiente al 70,9\% de los pacientes con niveles de presión arterial menor a 140/90 (Tabla 1).

Tabla 1. Niveles de Presión Arterial Sistólica y Diastólica. Modelo tomado de Duarte A. y Becerra N. (20)

\begin{tabular}{lccccccc}
\hline \hline PAS-PAD & $<80$ & $\mathbf{8 0 - 8 4}$ & $\mathbf{8 5 - 8 9}$ & $\mathbf{9 0 - 9 9}$ & $\mathbf{1 0 0 - 1 0 9}$ & $\mathbf{1 1 0}$ & TOTAL \\
\hline$<120$ & 4 & 2 & 0 & 0 & 0 & 0 & 6 \\
$120-129$ & 1 & 20 & 0 & 1 & 0 & 0 & 22 \\
$130-139$ & 2 & 7 & 0 & 7 & 0 & 0 & 16 \\
$140-159$ & 1 & 5 & 0 & 11 & 1 & 0 & 18 \\
$160-169$ & 0 & 0 & 0 & 0 & 0 & 0 & 0 \\
180 & 0 & 0 & 0 & 0 & 0 & 0 & 0 \\
TOTAL & 8 & 34 & 0 & 19 & 1 & 0 & 62 \\
\hline PAS/PAD $<140 / 90$ & & & & 44 & & $70,9 \%$ \\
PAS/PAD $>$ 140/90 & & & & 18 & & $29 \%$ \\
TOTAL & & & & & & & \\
\hline \hline
\end{tabular}

Referente a la función renal, el 60,2\% de los pacientes se encontraban controlados. El BUN estuvo en los rangos entre 9 y $19 \mathrm{mg} / \mathrm{dl}$ en ambos sexos y la Creatinina mostro un promedio dentro de los valores normales en las tres fechas de 0,5 a $1,1 \mathrm{mg} / \mathrm{dl}$ en mujeres y 0,6 a 1,2 $\mathrm{mg} / \mathrm{dl}$ en hombres. Aunque los cambios no fueron estadísticamente significativos con un Valor-P 0,5415, si es de importante relevancia clínica que los valores se mantuvieran dentro del rango normal en los cinco años de permanencia en el programa.

En relación al perfil lipídico, los niveles de HDL se mantuvieron por encima de $50 \mathrm{mg} / \mathrm{dl}$ en el $60 \%$ de los pacientes. Sin embargo, el LDL mostro en promedio un ascenso, de la primera a la segunda fecha, en la tercera fecha tuvo un descenso por debajo del valor de la primera. No obstante, los valores promedios de las tres fechas se encontraban por encima de $100 \mathrm{mg} / \mathrm{dl}$; su descenso a menos de $120 \mathrm{mg} / \mathrm{dl}$, no tuvo una significancia estadística, al mostrar un Valor-P de 0,2585 (Figura 1). De igual manera los triglicéridos reflejaron un descenso en la media de la primera a la tercera fecha de más de $40 \mathrm{mg} / \mathrm{dl}$, los resultados no fueron estadísticamente significativos con un valor $\mathrm{P}$ de 0,6075. Es importante resaltar, que los niveles iniciales se encontraban por encima de $150 \mathrm{mg} / \mathrm{dl}$ para el LDL y 200 $\mathrm{mg} / \mathrm{dl}$ de los triglicéridos (Figura 2).

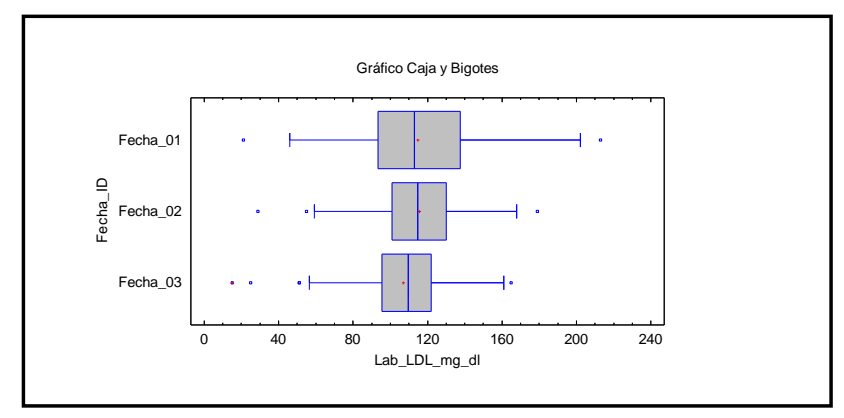

Figura 1. Comportamiento del LDL

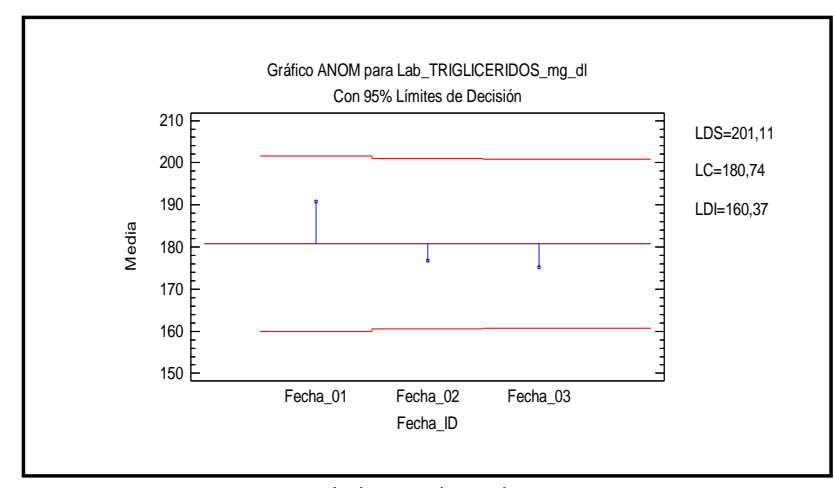

Figura 2. Comportamiento de los Trigliceridos

Fue identificada la disminución del peso y del índice 
de Masa Corporal, que en promedio, entre el primer y el último control disminuyeron 6 Kilogramos, conllevando a que el $71 \%$ de ellos estuvieran en el peso ideal. Así mismo el $60,5 \%$ disminuyeron y mantuvieron los niveles de glicemia en rango normal por debajo de los 100 $\mathrm{mg} / \mathrm{dl}$. Es por ello que al correlacional estas variables se encontró una relación positiva entre el IMC y la presión arterial diastólica, a mayor IMC, mayor acumulo de grasas en todo el organismo y en endotelio, causando ateroesclerosis, posterior mayor resistencia periférica y por ende se aumentan las presiones arteriales.
Otra correlación de importancia fue la del HDL con los niveles de presión sistólica y diastólica, que en el $60 \%$ de los pacientes se encontró por encima de $50 \mathrm{mg} / \mathrm{dl}$. Esta lipoproteína actúa como factor protector para enfermedades cardiovasculares, ayuda en el transporte de grasas fuera de las arterias, es por ello que entre más elevada este la HDL, mayor factor protector tiene, disminuyéndose de esa manera la resistencia periférica, por ende disminuye la presión arterial (Tabla 2).

Tabla 2. Correlación entre variables.

\begin{tabular}{|c|c|c|c|c|c|c|c|c|c|c|}
\hline & Peso_kg & TA_Sistolica & TA_Diastolica & IMC & Trigliceridos & $\mathrm{HDL}$ & LDL & Glicemia & BUN & Creatinina \\
\hline \multirow{2}{*}{ Peso_kg } & 1 & 0,0364 & 0,0859 & 0,7963 & $-0,0379$ & $-0,0366$ & $-0,0424$ & 0,0719 & 0,1180 & 0,1059 \\
\hline & 1 & 0,6649 & 0,3059 & 0,0000 & 0,6521 & 0,6635 & 0,6136 & 0,3919 & 0,1588 & 0,2063 \\
\hline \multirow{2}{*}{ IMC } & & & & 1 & 0,1041 & $-0,0509$ & 0,0677 & 0,1823 & 0,0824 & $-0,0021$ \\
\hline & & & & 1 & 0,2143 & 0,5446 & 0,4199 & 0,0288 & 0,3260 & 0,9805 \\
\hline \multirow{2}{*}{ HDL } & & & & & & 1 & $-0,3058$ & 0,0389 & 0,0103 & $-0,0985$ \\
\hline & & & & & & 1 & 0,0002 & 0,6436 & 0,9028 & 0,2402 \\
\hline
\end{tabular}

Los resultados anteriores coincide con las respuestas de los pacientes en las encuestas al afirmar: el $98 \%$ haber sentido cambios en su salud desde que se encuentran asistiendo al programa de HTA y el $98,4 \%$ indicó que la educación brindada por el personal de salud del programa lo ha llevado a cambiar su estilo de vida (Tabla 3).

Tabla 3. Cambios en la salud de los pacientes.

\begin{tabular}{cccc}
\hline \hline Pregunta & Nivel & Frec. Obs. & Frec. $\%$ \\
\hline Ha sentido usted cambios en su salud & Sí & 61 & $98.4 \%$ \\
desde que está asistiendo al programa & No & 1 & $1.6 \%$ \\
de H.T.A? & & 62 & $100.0 \%$ \\
TOTAL & &
\end{tabular}

Los pacientes haber modificado hábitos pocos saludables como la disminución del consumo de sal un 98\% y grasas Trans el 92\%; un $88,7 \%$ aprendieron a balancear los alimentos; el 53,2\% dan un mejor manejo al estrés; un 40,3\% disminuyó el sedentarismo; y, en menor proporción con un 3,6\% dejaron de fumar. También manifestaron el $97 \%$ de los pacientes cambios de su condición física, reflejado en el $72,6 \%$ de los pacientes que estaban en sobre peso lo bajaron; el $95,2 \%$ disminuyeron los problemas articulares de hombro y rodilla; y, el $79 \%$ se siente mejor con su apariencia física actual. El $100 \%$ de los pacientes indicaron sentirse satisfechos por la atención brindada por el equipo de salud (Figura 3).

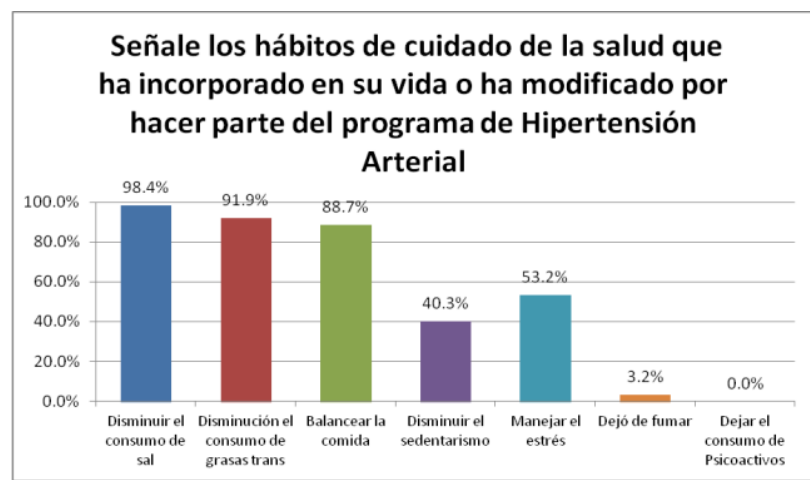

Figura 3. Incorporación de hábitos de auto-cuidado por los pacientes.

\section{DISCUSIÓN}

El presente estudio muestra una alta frecuencia de trabajadores con sobrepeso al compararla con la prevalencia general en Colombia que es del 34,6\% y similar a lo reportado por Martínez (40,1\%) (1) y Alonso (49\%) (15); este hallazgo unido a los demás factores de riesgo encontrados, específicamente un alto consumo de alcohol y patrones alimentarios inadecuados por el bajo consumo de frutas y verduras, colocan a la empresa en la necesidad de implementar medidas de promoción de la salud y prevención de la enfermedad.

En relación con el consumo de tabaco, el reporte fue muy bajo al relacionarlo con otros estudios, donde un $35 \%$ de los trabajadores encuestados eran fumadores activos (15).

Como sucede en otros estudios la inactividad física fue mayor durante el tiempo libre, entendiendo que la mayoría de los participantes del estudio son operarios existe una mayor probabilidad de ser activo físicamente durante el trabajo o el transporte (16); la evaluación de 
la práctica de actividad física está mediada por el dominio, considerado como las oportunidades de la vida cotidiana donde las personas puedan realizarla, por ello, el tipo de ocupación de la persona determina si se es activo en el ámbito laboral o en el tiempo libre.

Los participantes del presente estudio reportan mayores niveles de actividad por las actividades laborales que realizan relacionadas con el área de trabajo operario, que implican levantamiento de carga, manipulación de equipos, mantenimiento preventivo y correctivo de maquinaria entre otras actividades.

Este tipo de estudio es relevante porque evidencia el compromiso de la empresa en la detección precoz de los factores de riesgo cardiovasculares y por la posibilidad que los trabajadores acudan al sistema de salud toda vez que las personas en edad laboral solicitan consulta médica según las patologías percibidas (17).

Los programas de promoción de la salud en el sitio de trabajo pueden modificar factores de riesgo como inactividad física, tabaquismo, hipertensión, dislipidemia, mala alimentación, hiperglucemia y estrés psicológico elevado; y aunque los empleadores consideren que la tarea de mejorar la salud de sus empleados le corresponde a las instituciones de salud, debido a las jornadas de trabajo las personas no pueden asistir a los servicios de atención, el escenario laboral entonces se convertiría en una opción para fomentar estilos de vida saludable $(18,19)$.

El diseño de corte transversal en una limitación de esta investigación porque no permite establecer inferencias causales entre las variables estudiadas, aunque si la postulación de hipotesis causales que permitan plantear futuras investigaciones.

\section{REFERENCIAS BIBLIOGRÁFICAS}

1. Organización Mundial de la Salud. Información general sobre la Hipertensión Arterial en el mundo. 2013. [Citado 2014 julio 10] Disponible: http://apps.who.int/iris/bitstream/10665/87679/1/ WHO_DCO_WHD_2013.2_spa.pdf

2. Rubinstein Adolfo, "Importancia del adecuado control y manejo de la hipertensión arterial", en: Atención Primaria de la Salud Boletín PROAPSREMEDIAR, Buenos Aires, año 1, nㅇ2, 2003, p9.

3. Douglas D. La prehipertensión podría indicar un mayor riesgo cardiovascular. Am J Hipertensión. 2007; 20: 483-491

4. Achury-Saldaña, D., Rodríguez, S. M., Achury-Beltrán, L. F., Padilla-Velasco, M. P., et al. (2013). Efecto de un plan educativo en la capacidad de agencia de autocuidado del paciente con hipertensión arterial en una institución de segundo nivel. Aquichan. Vol. 13, No. 3, 363-372.

5. Pérez-Cuevas $R$, Reyes Morales $H$, Doubova SV, Zepeda Arias M, Díaz Rodríguez G, Peña Valdovinos $A$, et al. Atención integral de pacientes diabéticos e hipertensos con participación de enfermeras en medicina familiar. Rev Panam Salud Pública. 2009;26 (6):511-7

6. Segarra Edgar. Encalada L, García J L. Manejo de la hipertensión arterial desde los servicios de atención primaria. Revista semestral de la DIUC 57 MASKANA, Vol. 2, No. 2, 2011. Facultad de Ciencias Médicas, Universidad de Cuenca, Ecuador.

1. 7. Hacihasanoglu R, Gözüm S. The effect of patient education and home monitoring on medication compliance, hypertension management, healthy lifestyle behaviors and BMI in a primary health care setting. Journal of Clinical Nursing. 011;(20):692-705.

7. Resendiz E., Aguilera P. y Rocher M.E. (2010). Cita a: Barquera-F S, Barquera S, García E, GonzálezVillalpando, C, Hernández-A M, Lonngi G et ál.. En: Estilos de vida e índice de masa corporal de una población de adultos del sur de Tamaulipas México. Aquichan. Vol. 10, No. 3, $244-252$.

8. Paffenbarger R. Physical activity and hypertension. An epidemiological view. Annals Med 1991; 23: 319.

9. Fraser G. Preventive Cardiology. New York: Oxford University Press; 1986.

10. Stamler R., Stamler J., Gosch F., Civinelli J., Fishman J., Mckeever P. Primary prevention of hypertension by nutritional hygienic means. JAMA 1989; 262: 1.801-1.807. [Citado 2014 julio 28] Disponible: http://www.ncbi.nlm.nih.gov/pubmed/0002778913

11. OPS- Ministerio de Salud y protección social, Instituto Nacional de Salud. Indicadores básicos 2010. Situación de Salud en Colombia.

12. Ministerio de Salud y Protección Social. Sistema de monitoreo y evaluación al plan decenal de salud pública 2012-2021. Bogotá D.C., Colombia; 2013. [Citado 2014 agosto 25] Disponible: http://tic.uis.edu.co/ava/pluginfile.php/153493/mod _resource/content/1/Seguimiento\%20y\%20Evaluaci \%C3\%B3n\%20del\%20PDSP2012\%20-\%202021.pdf

13. Ministerio de Salud y Protección Social. Dimensión vida saludable y condiciones no transmisibles. Bogotá D.C., Colombia; 2013. [citado 2014 septiembre 25] Disponible: http://www.minsalud.gov.co/plandecenal/Document s/dimensiones/dimensionvidasaludablecondicionesno-transmisibles.pdf

14. Prado García H, Rojas Gómez I. Programa educativo 
sobre hipertensión arterial a los pacientes que acuden al club de hipertensos la sabanita. ciudad bolívar-estado bolívar. Universidad De Oriente, Escuela Cs De La Salud, Departamento de Enfermería. 2010. [Citado 2014 agosto 15] Disponible:

http://ri.biblioteca.udo.edu.ve/bitstream/123456789 /2244/1/47\%20Tesis.\%20WG9\%20P896.pdf

15. Varela Arévalo M. El reto de evaluar la adherencia al tratamiento en la hipertensión arterial. Pensamiento Psicológico, vol. 7, No 14, 2010, pp. 127-140. [Citado 2014 agosto 19] Disponible: http://www.redalyc.org/pdf/801/80113673010.pdf

16. CARMELA. El Riesgo Cardiovascular en América Latina. 2010. [Citado 2014 junio 29] Disponible: www.interamericanheart.org/estudio-carmela

17. Bastida C. Asociación entre las capacidades de agencia de autocuidado y farmacológico y no farmacológico en personas con alguna condición coronaria. En Enfermería Vol. XXV (2).No 65-67 2007. [27 de julio de 2013] Disponible en: www.indexf. com/rawe/252/r6575.php

18. Arrieta Cárdenas C. Impacto de un programa de prevención de enfermedades cardiovasculares en pacientes hipertensos y diabéticos. Facultad de medicina. Universidad autónoma de Bucaramanga. 2007. [Citado 2014 septiembre 6] Disponible: http://bdigital.ces.edu.co:8080/dspace/bitstream/12 3456789/140/1/IMPACT 1.PDF

19. Duarte A., Becerra N. valoración del impacto de de las actividades realizadas en la clínica de atención integral a pacientes diabéticos e hipertensos del Hospital Universitario de San Ignacio. 2009. [Citado 2014 junio 10] Disponible: http://med.javeriana.edu.co/publi/vniversitas/serial/ v50n2/2-VALORACION.pdf

20. Tuesca Molina R., Navarro Lechuga E., Peñuela M., Vargas R. Salud y medio ambiente en una población del Caribe colombiano: encuesta en Soledad (Atlántico). Barranquilla. Universidad del Norte. 2011. [Citado 2014 agosto 15] Disponible: http://www.uninorte.edu.co/documents/72553/6d3 b4173-9318-48d4-949a-a4ace2ce06c4

21. Wolf-Maier K, Cooper RS, Kramer H, Banegas JR, Giampaoli S, Joffres $M R$, et al. Hypertension treatment and control in five European countries, Canada, and the United States. Hypertension. 2004;43:10. [Citado 2014 septiembre 18] Disponible: http://www.ncbi.nlm.nih.gov/pubmed/14638619

22. European Society of Hypertension (ESH) and European Society of Cardiology (ESC) Task Force for the Management of Arterial Hypertension. (2007). 2007 Guidelines for the management of essential hypertension of the European Society of Hypertension (ESH) and of the European Society of Cardiology (ESC). Journal of Hypertension, 25, 10051187

23. OPS/OMS. Informe del Grupo Asesor para Hipertensión. Was־hington: OPS; 2001.

24. Ymiracy NS. Práctica educativa en un grupo de hipertensos. Colombia Médica. 1997; Vol. 28:130135. [Citado 2014 julio 25] Disponible: http://www.redalyc.org/pdf/283/28328305.pdf

25. Pinson AG. Educación del paciente cardiovascular en los programas de rehabilitación cardiaca. Medigrafic. 2000; 8(4):25- 30. [Citado 2014 septiembre 8] Disponible:

http://www.medigraphic.com/pdfs/enfe/en2000/en001-4e.pdf

26. Viera AJ, Kshirsagar AV, Hinderliter AL. Lifestyle modifications to lower or control high blood pressure: Is advice associated with action? The behavioral risk factor surveillance survey. The Journal of Clinical Hypertension. 2008;10(2):105-111.

27. Melano-Carranza E, La sses Ojeda LA, Avila-Funes JA. Factores asociados con la hipertensión no tratada en los adultos mayo-res: resultados del estudio nacional sobre salud y envejecimien-to, 2001. Rev Panam Salud Pública 2008; 23, (5); 395-302.

28. Lyngcoln, A., Taylor, N., Pizzari, T. \& Bakus, K.. The Relationship between Adherence to Hand Therapy and Short-term Outcome after Distal Radius Fracture. Journal of Hand Therapy. 2005; 18(1), 2.

29. Holguín L., Correa D., Arrivillaga M., Cáceres D., Varela M. Adherencia al tratamiento de hipertensión arterial: efectividad de un programa de intervención biopsicosocial. Univ. Psychol. v.5 n.3 Bogotá dez. 2006 [Citado 2014 septiembre 2] Disponible: http://pepsic.bvsalud.org/scielo.php?pid=S165792672006000300009\&script=sci_arttext

30. Marco para la Evaluación de Programas en Salud Pública. [internet]. 1999;48: RR-11 [Citado 2013 noviembre 16]. Disponible: http://www.cdc.gov/eval/index.htm.

Las opiniones que contenga este artículo son de su(s) autor(es) y no necesariamente representan la opinión oficial de la Universidad Simón Bolívar, la Revista Ciencia e Innovación en Salud o de sus autoridades. 